BMC

Plant Biology

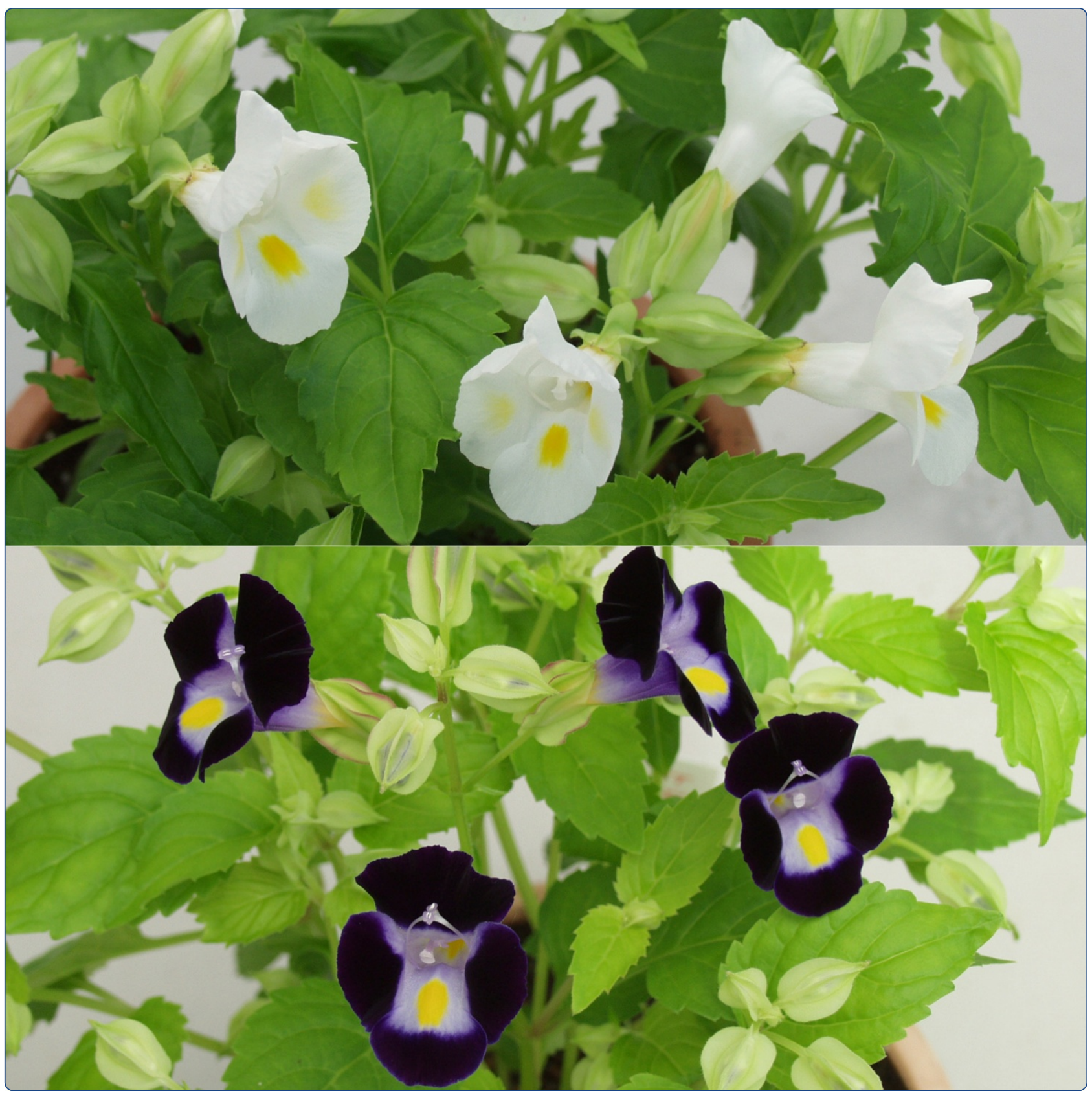

\title{
Molecular characterization of mutations in white-flowered torenia plants
}

Nishihara et al.

() Biomed Central 


\title{
Molecular characterization of mutations in white-flowered torenia plants
}

\author{
Masahiro Nishihara ${ }^{1 *}$, Eri Yamada ${ }^{1}$, Misa Saito ${ }^{1}$, Kohei Fujita ${ }^{1}$, Hideyuki Takahashi ${ }^{1}$ and Takashi Nakatsuka ${ }^{2}$
}

\begin{abstract}
Background: Torenia (Torenia fournieri Lind.) is a model plant increasingly exploited in studies in various disciplines, including plant engineering, biochemistry, physiology, and ecology. Additionally, cultivars with different flower colors have been bred and made commercially available. Flower color in torenia is mainly attributed to the accumulation of anthocyanins, but the molecular mechanisms inducing flower color mutations in torenia have not been well elucidated. In this study, we therefore attempted to identify the cause of white coloration in torenia by comparing the white-flowered cultivar Crown White (CrW) with Crown Violet (CrV), a violet-flowered variety.

Results: In an expression analysis, no flavanone 3-hydroxylase (TfF3H) transcript accumulation was detected in CrW petals. Sequence analyses revealed that a novel long terminal repeat (LTR)-type retrotransposable element, designated as TORE1 (Torenia retrotransposon 1), is inserted into the 5'-upstream region of the TfF $3 \mathrm{H}$ gene in $\mathrm{CrW}$. A transient expression assay using torenia F3H promoters with or without TORE1 insertion showed that the TORE1 insertion substantially suppressed $\mathrm{F} 3 \mathrm{H}$ promoter activity, suggesting that this insertion is responsible for the absence of $\mathrm{F} 3 \mathrm{H}$ transcripts in white petals. Furthermore, a transformation experiment demonstrated that the introduction of a foreign gentian F3H CDNA, GtF3H, into $\mathrm{CrW}$ was able to recover pink-flower pigmentation, indicating that $\mathrm{F} 3 \mathrm{H}$ deficiency is indeed the cause of the colorless flower phenotype in CrW. Detailed sequence analysis also identified deletion mutations in flavonoid $3^{\prime}$-hydroxylase (TfF ${ }^{\prime} H$ ) and flavonoid $3^{\prime}, 5^{\prime}$ - hydroxylase (TfF3' $5^{\prime} H$ ) genes, but these were not directly responsible for white coloration in this cultivar.
\end{abstract}

Conclusions: Taken together, a novel retrotransposable element, TORE1, inserted into the F3H 5'-upstream region is the cause of deficient $F 3 H$ transcripts in white-flowered torenia, thereby leading to reduced petal anthocyanin levels. This is the first report of a retrotransposable element involved in flower color mutation in the genus Torenia.

Keywords: Torenia fournieri, F3H, Muation, LTR-type retrotransposon, White flower

\section{Background}

Natural or spontaneous mutations, which accelerate evolution in living organisms, have a number of causes. In flowering plants, flower color mutations occur widely in nature, and floricultural plants in a variety of flower colors have been produced through artificial selection of this natural variability. Flavonoids, such as flavones, aurones, flavonols, and anthocyanins, are the most important plant pigments associated with flower coloration. The flavonoid biosynthetic pathway is one of the most extensively studied pathways in plant specialized metabolism [1]. The biosynthetic genes necessary for biosynthesis of colored anthocyanins

\footnotetext{
* Correspondence: mnishiha@ibrc.or.jp

'Iwate Biotechnology Research Center, Narita 22-174-4, Kitakami, Iwate 024-0003, Japan

Full list of author information is available at the end of the article
}

have been especially well studied in many plant species, including, Arabidopsis, snapdragon, petunia, grape, and maize, and have become targets for molecular breeding [1-4]. In addition to flower coloration, studies of anthocyanin-based coloration and associated mutations in fruits, seeds, and other organs have provided information helpful for elucidation of flavonoid biosynthesis-related genes. In particular, recent molecular biological investigations have revealed the basic mechanisms generating these mutations and their effects on pigmentation at the molecular level in different plant species.

Torenia (Torenia fournieri Lind., also known as wishbone flower) is a perennial plant widely used as a bedding flower from early spring through summer. Cultivars in different flower colors, such as white, blue, and pink, have been generated by conventional breeding and are now commercially 
available. Because of its various merits in regard to molecular analyses (reviewed in $[5,6]$ ), the species is proposed as a potential new model flower to study a wide range of floral traits. Torenia fournieri and T. hybrida (T. fournieri $\times T$. concolor) are frequently used in transgenic studies of various subjects such as flower color [7-9], anthocyanin synthesis [10], chlorophyll degradation [10], flower shape [11-14], and fertilization [15]. Among studied characteristics, flower color is one of the most targeted traits in molecular investigations, as flower color modification is of strong interest from both basic and applied research perspectives. Torenia flower pigments are composed of flavonoid anthocyanins. Chemical analysis has revealed the anthocyanin components of $T$. fournieri 'Crown Violet' [7] and T. hybrida 'Summerwave Blue' [16]. Using these cultivars, flower colors ranging from the original violet to white, pink, and yellow have been successfully produced by genetic engineering of flavonoid biosynthetic genes [7-9,16-19].

As mentioned above, variously flower-colored torenia cultivars and lines have been produced by both conventional and molecular methods, but the origins of color mutations in the breeding materials are largely unknown. Because they are easily recognizable by eye and hence good plant research materials, flower color mutations have been studied extensively in species such as petunia [20,21], snapdragon [22,23], and morning glory $[24,25]$. For example, the variegated-flower morning glory mutation is caused by the insertion of the Tpn1 transposable element into the dihydroflavonol 4-reductase (DFR) gene [26], and white-flowered morning glory is derived from insertions of Tpn1-related DNA transposable elements into an intron of the chalcone synthase $(\mathrm{CHS})$ gene [27]. Red-flowered phenotypes in commercial petunias have been attributed to transposon insertion mutations of the $F 3^{\prime} 5^{\prime} H$ gene [28]. Similarly, various mutations related to flower pigmentation have been studied in carnation [29-31], with class I, class II, and other transposable elements implicated in the color changes. We have also identified several DNA- and RNA-type transposable elements in white- or pinkflowered mutants in Japanese gentian flowers [32-34]. With respect to torenia, little is known regarding the source of flower color mutations, with one exception: Nishijima et al. [35] recently reported that a 'flecked' mutant, bearing variegated flowers, originated through the insertion of an Enhancer/Suppressor-Mutator (En/ Spm)-like transposon ( $T t f 1)$ into the intron of the TfMYB1 transcription factor gene. The causal agents of flower color mutations in other torenia cultivars have not yet been identified.

In the present study, we uncovered and analyzed mutations in a white-flowered torenia through comparison with a violet-flowered cultivar. We first identified a novel retrotransposable element, designated TORE1, in torenia based on a molecular biological approach. Using both transient and stable transformation experiments, we then confirmed that the insertion of TORE1 into the flavanone 3-hydroxylase $(F 3 H)$ promoter region contributes to the white-flowered phenotype. We found two additional mutations in this cultivar and considered their association with white coloration. This is the first known identification of a retrotransposable element in the genus Torenia.

\section{Results}

\section{Analysis of flavonoid components in torenia flowers}

As shown in Figure 1A and 1B, T. fournieri cultivars Crown Violet $(\mathrm{CrV})$ and Crown White $(\mathrm{CrW})$ have violet and white flower petals, respectively. Spectral profiles of $0.1 \%$ $\mathrm{HCl}$-methanol extracts of each flower are shown in Figure $1 \mathrm{C}$. In the visible light region, $\mathrm{CrV}$ had a maximum absorption wavelength at $530 \mathrm{~nm}$ corresponding to anthocyanin pigments; in contrast, $\mathrm{CrW}$ had no detectable peak in the visible spectrum, indicating that no anthocyanins accumulated in this white-flowered cultivar. UV absorption spectra revealed that $\mathrm{CrW}$ and $\mathrm{CrV}$ had maximum absorption peaks at 346 and $333 \mathrm{~nm}$, respectively. To identify the flavone components accumulating in each cultivar, acid hydrolysis was performed followed by HPLC analysis. The flavone aglycones identified in $\mathrm{CrV}$, as described previously [7], were apigenin and luteolin, whereas $\mathrm{CrW}$ contained only apigenin. Total levels of flavone derivatives were 1.8 times higher in $\mathrm{CrW}$ than in $\mathrm{CrV}$ (Figure 1D). These results suggest one possibility that the hydroxylation activity of flavonoid B-rings is deficient in CrW.

\section{Expression analysis of flavonoid biosynthetic genes in torenia flowers}

We attempted to screen the mutated genes by means of an expression analysis of torenia flower petals. As shown in Additional file 1: Table S1, we designed primers based on flavonoid biosynthetic genes of T. hybrida, because full-length sequences, except for chalcone isomerase (CHI), are available for this hybrid cultivar. Using these primers, which were designed for nearly full-length amplification, RT-PCR analysis was performed on the flavonoid biosynthesis-related genes CHS, CHI, F3H, DFR, anthocyanidin synthase (ANS), $F 3^{\prime} H, F 3^{\prime} 5^{\prime} H$, anthocyanin 5-O-glucosyltransferase (5GT), and flavone synthase II (FNSII) (Figure 2A). Fragments of the expected length were amplified from each gene, and similar expressions were observed between the two cultivars for all genes except for F3H. Expressions of TfMYB1 and TfbHLH1, recently identified as transcription factor genes regulating anthocyanin biosynthesis in torenia flowers [35], also did not differ significantly between the two cultivars (Additional file 2: Figure S1). Furthermore, attempts to amplify partial fragments of $F 3 H$ using several different 


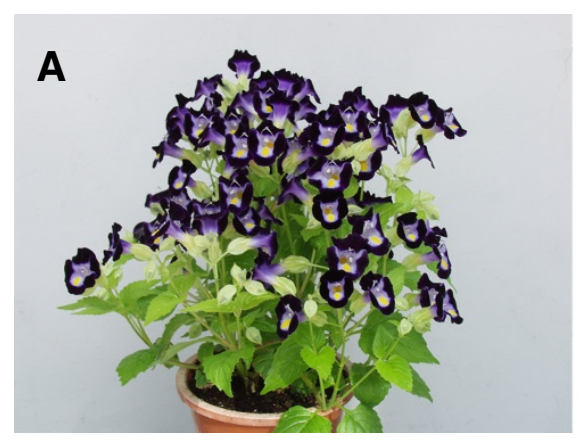

C

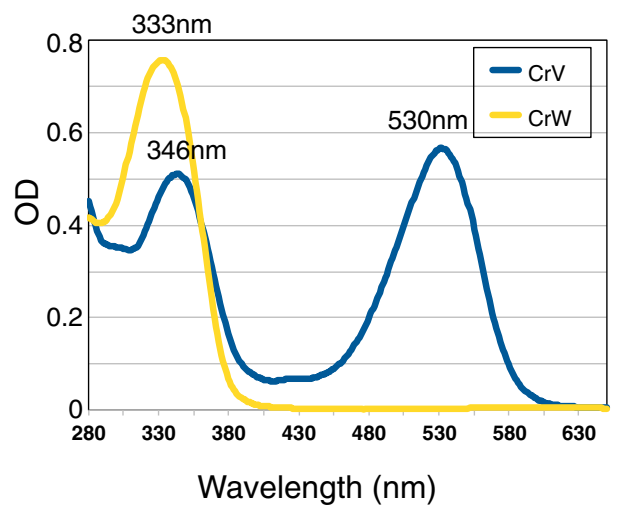

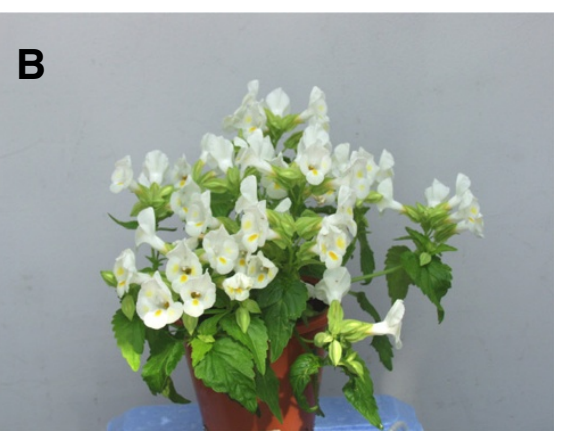

D

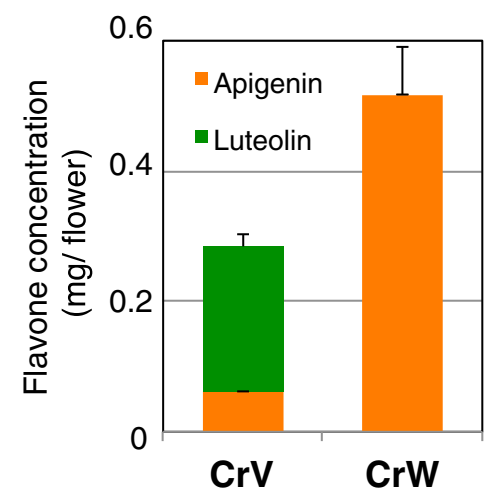

Figure 1 Torenia cultivars used in this study. (A) Crown Violet. (B) Crown White. (C) Absorbance spectrum of $0.1 \% \mathrm{HCl}-\mathrm{methanol}$ extracts of flower petals. (D) Flavone aglycone concentrations of CrV and CrW determined by HPLC analysis. Averages of four flowers \pm standard deviations are shown.

primers were unsuccessful (data not shown), indicating that $\mathrm{F} 3 \mathrm{H}$ expression was completely suppressed in $\mathrm{CrW}$. To elucidate the expressions of $F 3 H, F 3^{\prime} H$, and $F 3^{\prime} 5^{\prime} H$, we next performed northern blot analyses using the same RNAs (Figure 2B). RT-PCR results. With respect to the other two hydroxylases, F3' $H$ expression remained unchanged and $F 3^{\prime} 5^{\prime} H$ expression was slightly decreased. No visible band shifts were detected, indicating that no large indels were present in these two genes.

\section{Identification of genomic and CDNA sequences of three flavonoid biosynthetic genes in torenia plants}

Based on the results of the pigment and expression analyses, we speculated that three genes, $F 3 H, F 3^{\prime} H$, and $F 3^{\prime} 5^{\prime} \mathrm{H}$, were causally related to the $\mathrm{CrW}$ flower color mutation. We therefore compared genomic sequences of these three genes between the two cultivars. We generated DNA fragments that spanned start and stop codons using genomic PCR, and analyzed the sequences. F3H was identical between $\mathrm{CrV}$ and $\mathrm{CrW}$, whereas deletion mutations were present in $F 3^{\prime} H$ and $F 3^{\prime} 5^{\prime} H$ genomic sequences in both $\mathrm{CrV}$ and $\mathrm{CrW}$ (Additional file 2: Figure S2). In particular, a 12-bp deletion was observed in exon 3 of the $\mathrm{CrW} F 3^{\prime} H$ gene, presumably lacking four amino acid residues (Additional file 2: Figure S2A).
The $F 3^{\prime} 5^{\prime} \mathrm{H}$ gene from $\mathrm{CrW}$ also had a single-base (cytosine) deletion in exon 1, resulting in a frame shift (Additional file 2: Figure S2B). These mutated $F 3^{\prime} H$ and $F 3^{\prime} 5^{\prime} \mathrm{H}$ sequences were also present in $\mathrm{CrV}$, indicating that $\mathrm{CrV}$ is heterozygous for both gene mutations.

\section{Isolation of the $5^{\prime}$-upstream region of the $\mathrm{F} 3 \mathrm{H}$ gene from torenia plants}

Because no mutation was found in the $F 3 H$ gene, including coding exon and intron sequences, we suspected that $\mathrm{CrW}$ might have a specific mutation in the $5^{\prime}$-upstream region of $\mathrm{F} 3 \mathrm{H}$. To clone the $\mathrm{F} 3 \mathrm{H}$ upstream region, an inverse PCR was therefore performed. A 3,898-bp sequence upstream of the start codon was identified in CrV (accession no. AB902919) and found to contain cis-motifs such as a P-recognition element, an ACGT-containing element, and several MYB-related elements (Figure 3). Interestingly, the F3H upstream region of CrW (accession no. AB902920) contained a long 3,464-bp insertion at the -249th position prior to the transcription start site (Figure 3). This insertion had the features of an LTR-type retrotransposon, and was accordingly designated as Torenia retrotransposon 1 (TORE1). Our analysis revealed that TORE1 contains a completely identical pair of LTRs (560 bp) that start with TG and end with $\mathrm{CA}-\mathrm{a}$ typical canonical sequence- 


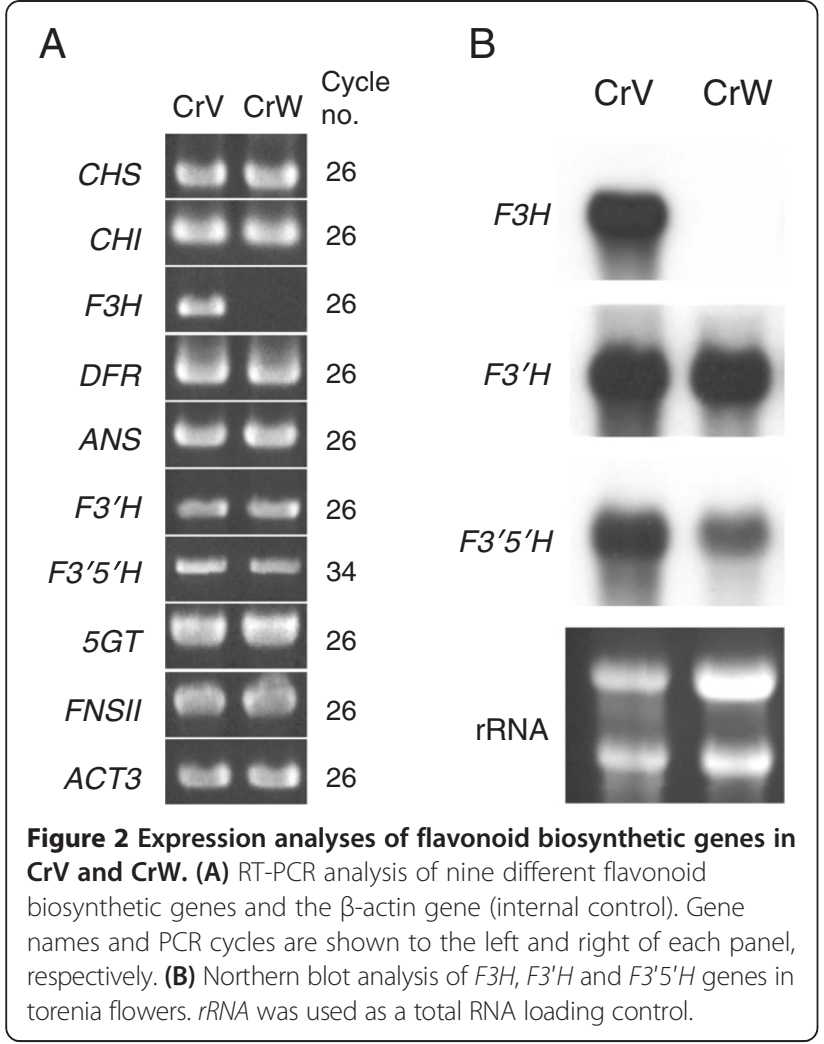

flanked by 5-bp direct repeats of the TfF3H sequence. TORE1 also contains a single open reading frame (ORF) encoding a putative gag-pol polyprotein of 605 amino acid residues. A blastp search revealed that the protein has partial homology to hypothetical proteins of Vitis vinifera, the Copia-like LTR Rider of Solanum lycopersicum [36], and a putative gag-pol polyprotein of Citrus sinensis [37]. The protein contains gag and protease, but seemingly lacks typical pol proteins such as integrase, reverse transcriptase, and RNaseH. As shown in Additional file 2: Figure S3, a primer binding site (PBS) and a polypurine tract (PPT) are also present in TORE1. In addition to the complete length of TORE1, genomic PCR amplification of CrW yielded a $5^{\prime}$-upstream fragment of $F 3 H$ containing only a solo LTR (Additional file 2: Figure S4). In CrV, the amplified $5^{\prime}$-upstream region of $F 3 H$ within $3.9 \mathrm{~kb}$ of the start codon did not contain TORE1 or TORE1-related sequences.

\section{Southern blot analysis of three flavonoid biosynthetic genes and TORE 1 in torenia}

To determine the status of the three mutated genes and the retrotransposable element TORE1 in each torenia cultivar, Southern blot analyses were performed (Figure 4; Additional file 2: Figure S5). Digestion with EcoR I and $X b a \mathrm{I}$, which do not cleave the $\mathrm{F} 3 \mathrm{H}$ gene containing three exons and two introns, confirmed that the $\mathrm{FHH}$ gene is present as a single copy gene in the torenia genome (Figure 4A). Digestion with Hind III, which cleaves the second $F 3 H$ intron, generated two and four bands from $\mathrm{CrV}$ and $\mathrm{CrW}$, respectively. The larger bands generated from $\mathrm{CrW}$ may have been due to partial Hind III digestion within TORE1, as depicted in Additional file 2: Figure S6. Southern blot analyses, using as probes the 560-bp LTR (Figure 4B) or the gag-pol polyprotein sequence of 605 deduced amino acids (Figure 4C), uncovered several bands from all restriction enzymes, suggesting that TORE1 or related elements are ubiquitously present in the torenia genome. $F 3^{\prime} \mathrm{H}$ and $F 3^{\prime} 5^{\prime} \mathrm{H}$ genes were also found in single copies in the torenia genome (Additional file 2: Figure S5). Additional banding patterns were observed in $\mathrm{CrV}$, reflecting the heterozygosity of $\mathrm{CrV}$ mentioned above.

\section{Transient expression analysis for $\mathrm{F} 3 \mathrm{H}$ promoter activity}

Because the TORE1 insertion into the $5^{\prime}$-upstream region most likely has a deleterious effect on $\mathrm{F} 3 \mathrm{H}$ promoter activity, we measured this activity using a transient expression system in protoplasts of Arabidopsis

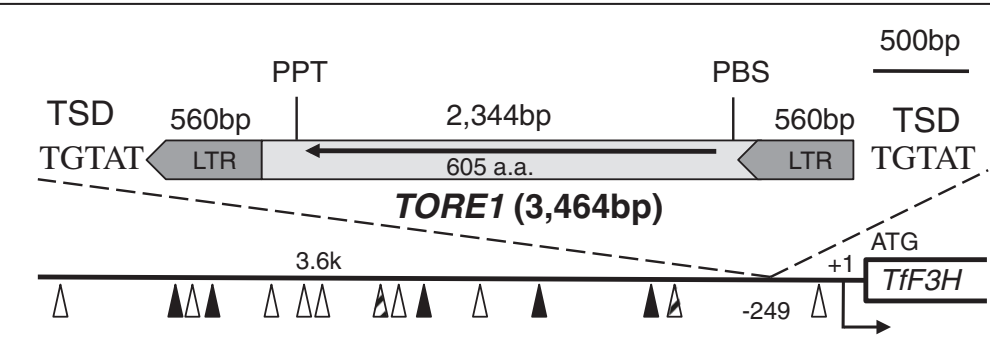

$\Delta \begin{gathered}\text { ACGT containing element } \\ (\text { CACGT) }\end{gathered}$ P recognition element $\quad \Delta_{\left(C^{\top} / A A C C\right)}^{\text {Binding sequence of vertebrate MYB protein }}$

3 Schematic diagram of the genomic structure of the $\mathrm{F} 3 \mathrm{H}$ gene in torenia. An insertion of TORE1 within the $5^{\prime}$-upstream region of F3H was identified in CrW. TORE1 has the features of long terminal repeat (LTR) retrotransposons, containing 5 bp of the target site duplication (TSD) and 560 bp of LTRs. A deduced open reading frame (ORF) is indicated by the black arrow. The primer binding site (PBS) and polypurine tract (PPT) are also shown. 


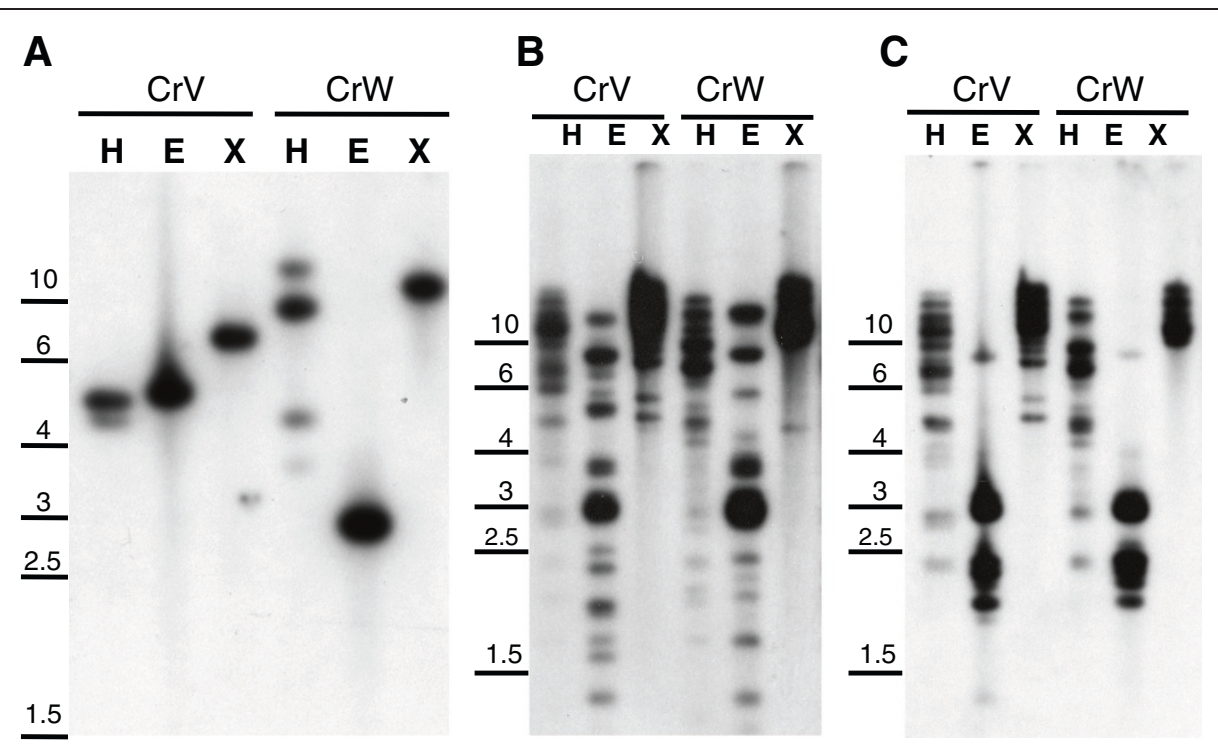

Figure 4 Southern blot analysis of $F 3 H$ and TORE1 in $\mathbf{C r V}$ and CrW. Total genomic DNAs were digested with Hind III (H), ECO RI (E), and Xba I $(X)$ and transferred to nylon membranes as described in Methods. Membranes were probed with DIG-labeled sequences of F3H (A), LTR (B), and gag-pol protein (C) from TORE1. DNA marker sizes (kbp) are shown.

suspension-cultured cells. Transcription factors GtMYB3 and GtbHLH1, isolated from gentian petals, are known to activate late-stage flavonoid biosynthetic genes $[33,38]$; we therefore used these two transcription factors as effectors in combination with different torenia $F 3 H$ promoter-firefly luciferase gene $(L U C)$ constructs, as shown in Figure 5. The 35Spro- Renilla luciferase gene $(R L U C)$ construct was also co-transformed as an internal standard. The approximately 1 -kbp $5^{\prime}$-upstream region of the torenia $\mathrm{F} 3 \mathrm{H}$ gene was used for this analysis. This 1 -kbp promoter was assigned a LUC/RLUC relative activity of 1 . Compared with the $1-\mathrm{kb}$ promoter, the $300-$ bp TfF $3 H$ promoter showed only $27.7 \%$ activity; the TORE1-inserted TfF3H promoter displayed an even greater activity reduction, down to $14.3 \%$. Insertion of the solo-LTR sequence alone also reduced promoter

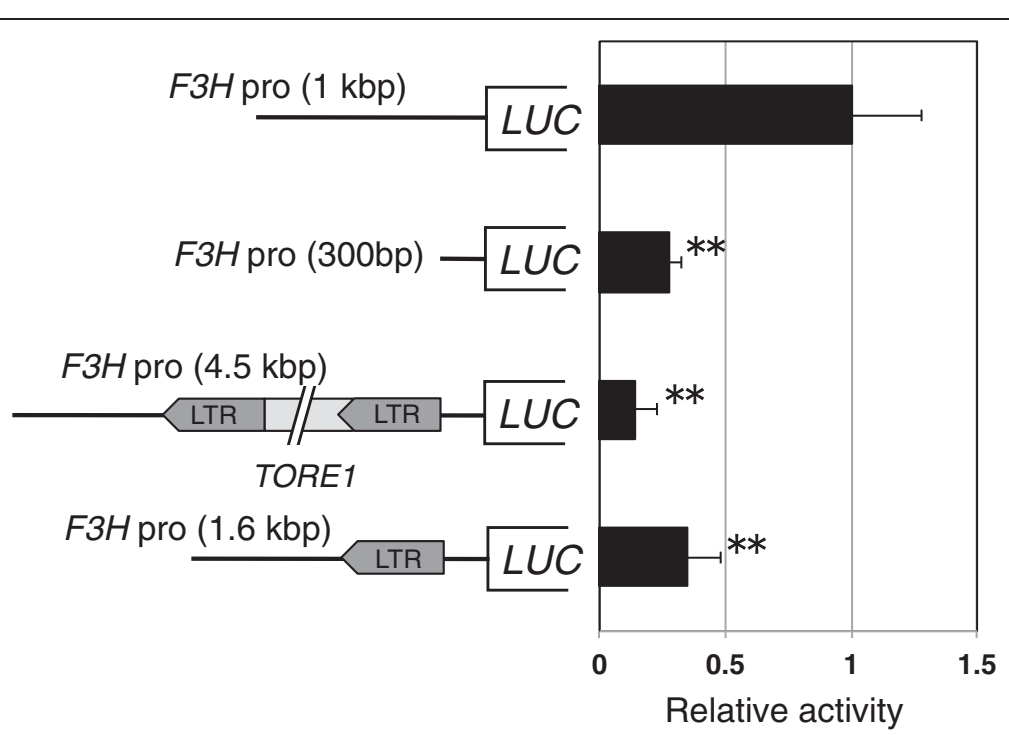

Figure 5 Transient expression assay for activation ability of $F 3 H$ promoters in Arabidopsis suspension cells. Suspension-cultured cells of Arabidopsis thaliana (T87 line) were used for this analysis. GTMYB3 and GtbHLH1 genes that can activate late-stage anthocyanin biosynthetic genes were co-introduced with various F3H promoters via PEG-mediated transformation. After $24 \mathrm{~h}$ culture, firefly luciferase (FLUC) activity was measured with a luminometer. A Renilla luciferase (RLUC)-driven Cauliflower mosaic virus (CaMV) 355 promoter was used for internal standardization of PEG infection. Relative activities (LUC/RLUC) are shown. Asterisks $\left(^{* *}\right)$ indicate significant differences between TfF3H pro (1 kbp) and other constructs $(P<0.01, t$-test $)$. 
activity (35.1\%). These data indicate that one or more cis-regulatory elements needed for $\mathrm{F} 3 \mathrm{H}$ expression are present in the upstream region $300 \mathrm{bp}$ above the translation start site, and that the TORE1 insertion definitely affects torenia $\mathrm{F} 3 \mathrm{H}$ promoter activity in vivo.

\section{Flower color complementation in $\mathrm{F} 3 \mathrm{H}$-overexpressed transgenic torenia plants}

To confirm whether the deficient $F 3 H$ expression in $\mathrm{CrW}$ is actually responsible for the white coloration, $\mathrm{CrW}$ was transformed with other functional $F 3 H$ cDNA. To produce transgenic torenia plants, we used a gentian $\mathrm{F} 3 \mathrm{H}$ gene that encodes a functional $\mathrm{F} 3 \mathrm{H}$ enzyme. $\mathrm{CrW}$ was transformed with the binary vector pSKan35SGtF3H (Figure 6A), and 46 transgenic torenia plants were regenerated and cultivated until flowering. Of these transgenic lines, 35 recovered pink-colored pigmentation in flowers, with some variability in the degree of pink color pigmentation; 30 lines had intense pink flowers, and five featured faint pink flowers. The upper lips of transgenic flowers were less pigmented than lower and lateral lips. Representative lines with intense pink (nos. 10 and 16) and faint pink (no. 4) flowers are shown in Figure 6B. Northern blot analysis confirmed the expression of the foreign gentian $F 3 H$ gene, indicating that the transformation was successful in torenia petals. Notably,

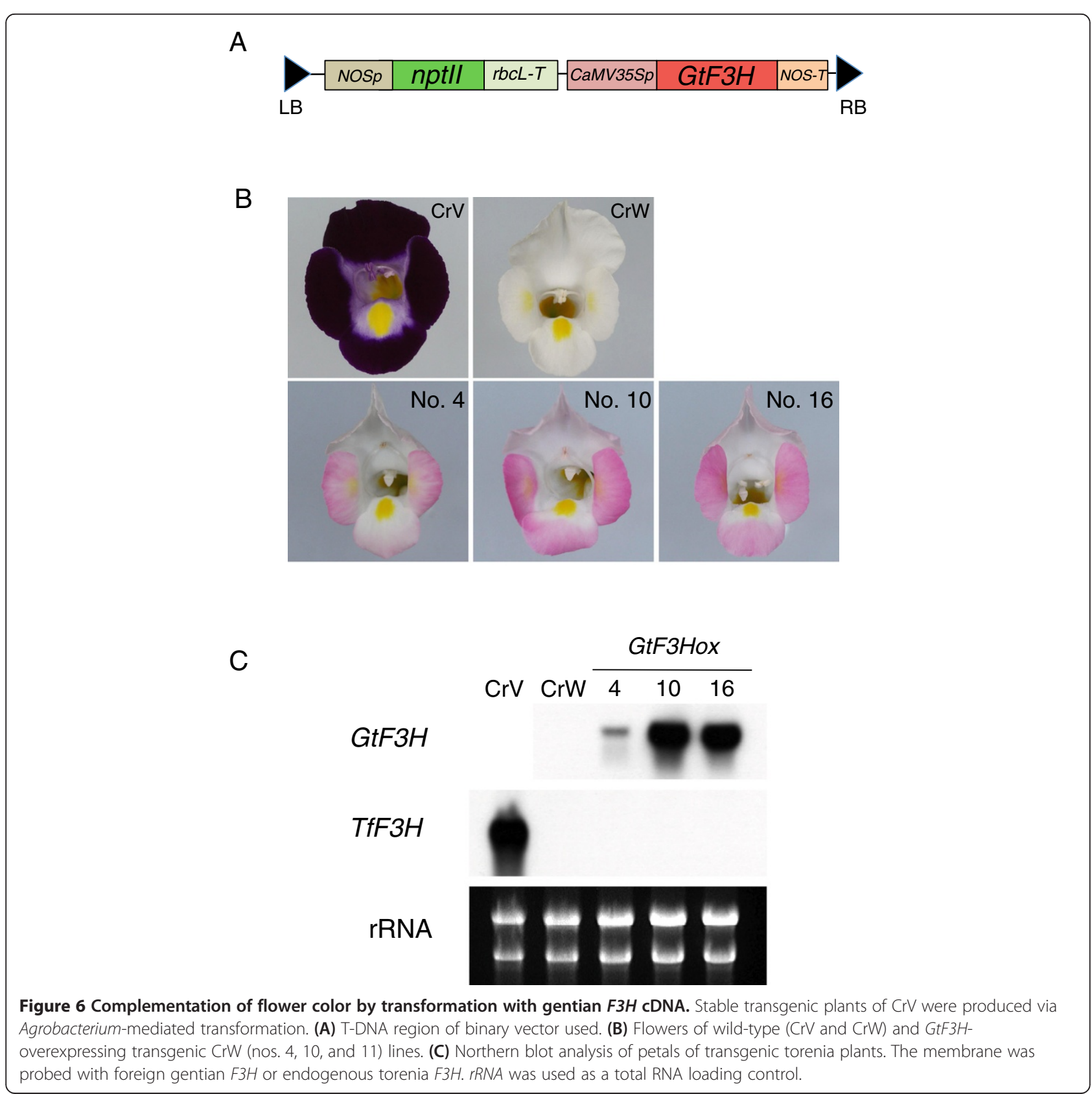


higher accumulation levels of GtF3H transcripts were observed in lines 10 and 16 than in line 4, consistent with recovered flower color intensities (Figure 6C). The endogenous torenia $\mathrm{FHH}$ gene remained suppressed in $\mathrm{CrW}$ and all three transgenic lines. HPLC analysis confirmed that the accumulated anthocyanidin in GtF3Hoverexpressed $\mathrm{CrW}$ was pelargonidin, not delphinidin or cyanidin (Additional file 2: Figure S7), indicating that the B-ring hydroxylation ability of anthocyanins was absent in CrW because of the lack of $F 3^{\prime} H$ and $F 3^{\prime} 5^{\prime} H$ activities.

\section{Discussion}

In this study, expression analysis of flavonoid biosynthetic genes revealed that only the expression of $\mathrm{F3H}$ was completely reduced in $\mathrm{CrW}$. F3H is a 2oxoglutarate-dependent dioxygenase that acts in the flavonoid biosynthetic pathway to hydroxylate flavanones to dihydroflavonols, which are common precursors for major classes of flavonoids, flavonols, catechins, anthocyanins, and proanthocyanidin in plants $[1,39]$. The gene encoding $\mathrm{FH} \mathrm{H}$ cDNA was first isolated from petunia [40], with homologous genes subsequently cloned in many plant species. $\mathrm{F} 3 \mathrm{H}$ gene mutations affecting color pigmentation have been reported in various plant species, including petunia [41], soybean [42], Japanese morning glory [24], and carnation [43]. Because anthocyanins are synthesized from dihydroflavonols, reduced F3H expression is the likely cause of the torenia $\mathrm{CrW}$ colorless flower phenotype.

Analysis of genomic structures did not uncover any mutations in either coding or intron regions of torenia $F 3 H$, but a long insertion (TORE1) was found in the $5^{\prime}-$ upstream region of $\mathrm{F3H}$ in $\mathrm{CrW}$. This insertion was identified as a retrotransposable element of the LTR subtype $[44,45]$. TORE1 has typical characteristics of LTRtype retrotransposons, namely 560-bp LTRs with 5 bps of target site duplication (TSD), a PBS, and a PPT, and encodes a partial gag-pol protein (Figure 3; Additional file 2: Figure S3). TORE1 may thus be a nonautonomous element derived from an originally autonomous one. Southern blot analysis also indicated the presence of TORE1-like elements in the torenia genome.

Transient expression analysis using various constructs of the $F 3 H$ promoter revealed that the TORE1 insertion is indeed involved in reduced promoter activity (Figure 5). For this analysis, we used the heterologous transcription factor genes GtMYB3 and GtbHLH1, which regulate flavonoid biosynthesis in gentian flowers. These two genes were chosen because they are well-characterized and constitute a reliable transient assay system established in our previous studies [33,38]. Although torenia endogenous TfMYB1 and $T f b H L H 1$ genes that are probably responsible for regulation of anthocyanin biosynthesis have been recently isolated, the evidence for TfMYB1-TfbHLH1 interaction leading to activation of flavonoid biosynthetic genes remains inconclusive. Further analysis is therefore required to clarify the effects of TfMYB1 and TfbHLH1 transcription factor genes on activation of the torenia $\mathrm{F} 3 \mathrm{H}$ promoter.

Many examples of transposable elements affecting gene expression have been noted in various organisms, with different classes of active transposons observed in genomes of eukaryotes including plants $[46,47]$. Transposable elements have been confirmed to contribute to plant evolution and diversity [48]. Retrotransposon or retrotransposon-like sequences, in particular, are ubiquitous components of plant genomes, and their impacts on plant genome structure and function have also been revealed (reviewed in [49]). Transposon insertions have been found in all types of genomic sequences, including coding (exon) and noncoding (intron, UTR, upstream, and downstream) regions. In a previous study, an $E n / S p m$-like transposon ( $T t f 1$ ) insertion in the TfMYB1 second intron was found to cause a mutant torenia phenotype by reducing expressions of flavonoid biosynthetic genes, including CHS, F3H, DFR, ANS, and UDP-glucose: flavonoid glucosyltransferase (UFGT) [35]. In our case, the insertion of TORE1 completely suppressed $F 3 H$ expression, as shown by RT-PCR and northern blot analysis. Transposable element insertions often induce epigenetic changes, such as DNA methylation, that cause strong gene silencing $[47,50]$; this fact suggests that TORE1 may reduce $F 3 H$ expression through a similar silencing mechanism. In fact, Southern blot analysis of $\mathrm{CrW}$ genomic DNA digested with Hind III revealed some larger bands when probed with $F 3 H$. Hind III is sensitive to cytosine methylation within the recognition site, and slowly cleaves hemimethylated AAG ${ }^{\mathrm{m} 5}$ CTT [51]. Partial digestion with Hind III due to the effect of de novo methylation has also been reported in transgenic pea plants showing inducible co-suppression of the transgene after virus infection [52]. Such an epigenetic change probably contributes, along with disruption of the $\mathrm{F} 3 \mathrm{H}$ promoter, to the reduction in $\mathrm{F} 3 \mathrm{H}$ promoter activity. Further studies using methylation-sensitive restriction enzymes or bisulfite sequencing would confirm this hypothesis.

Detailed examination of the $5^{\prime}$-upstream region of the F3H promoter in CrW genomic DNA revealed the presence of a solo-LTR insertion in addition to the fulllength TORE1 insertion (Figure 3; Additional file 2: Figure S4). Southern blotting generated a banding pattern inconsistent with the presence of the solo LTR in the $F 3 H$ promoter, indicating that the TORE1-inserted F3H and solo LTR-inserted F3H are not allelic. The solo LTR is probably derived from a recombination between LTRs within TORE1 in certain cells. As an example, the formation of solo LTRs through unequal homologous recombination of two LTRs has been previously reported in rice [53]. Based on the presence of solo LTRs and variously truncated fragments in plant genomes, unequal 
homologous recombination and illegitimate recombination have been inferred to drive genome size decreases in Arabidopsis [54] and rice [55]. CrW sometimes shows faint pink-recovered sections in flower petals (Additional file 2: Figure S8). A TORE1 excision event is probably not involved in this recovery, however, as class I transposons move by a "copy and paste" process. In the transient expression assay, the solo-LTR insertion was less effective in reducing promoter activity than was the full TORE1 insertion (Figure 5). This result suggests that the advent of the solo LTR is responsible for the partially recovered phenotype. Further studies are needed to determine the mechanism and developmental timing of this TORE1 recombination event in the $F 3 H$ promoter of $\mathrm{CrW}$ torenia plants.

The white coloration of $\mathrm{CrW}$ can clearly be attributed to the $F 3 H$ mutation, a conclusion confirmed by the complementation study (Figure 6). This finding is further supported by the fact that knockdown of torenia $F 3 H$ by RNAi produces white-flowered torenia plants in the blue-flowered cultivar Summerwave Blue [19]. Nevertheless, mutations were also found in $F 3^{\prime} H$ and $F 3^{\prime} 5^{\prime} \mathrm{H}$ gene sequences in $\mathrm{CrW}$ (Additional file 2: Figure S2). Deficiency of flavonoid B-ring hydroxylation activities is suggested by two observations: 1) apigenin was the only flavone accumulating in $\mathrm{CrW}$ petals (Figure 1) and 2) F3H-overexpressing transgenic $\mathrm{CrW}$ lines accumulated pelargonidin derivatives, not delphinidin derivatives (Additional file 2: Figure S7). $F 3^{\prime} H$ and $F 3^{\prime} 5^{\prime} H$ genes in $\mathrm{CrW}$ have deletion mutations that should affect enzyme activities. In particular, the single-base deletion in exon 1 of $F 3^{\prime} 5^{\prime} H$ results in a frameshift and subsequent premature termination of the enzyme (Additional file 2: Figure S2). Such mutations are frequently observed in other plants. In soybean, for example, single-base deletions in $F 3^{\prime} H$ and flavonol synthase ( $\left.F L S\right)$ are associated respectively with gray pubescence color [56] and magenta flower color [57]. A 4-bp insertion mutation in UDP-glucose:anthocyanidin 3-O-glucoside-2"-O-glucosyltransferase (3GGT) induces a dusky flower color in morning glory [58]. In three morning glory species, a single $\mathrm{C}$ to $\mathrm{T}$ base transition, a single $\mathrm{T}$ insertion, and insertion of the transposable element Tip201 in $F 3^{\prime} H$ are reportedly involved in reddish flowers [59]. In the case of $\mathrm{CrW}$, the 12-bp deletion in exon 3 of $F 3^{\prime} H$ did not cause a frameshift, but apparently resulted in the absence of four amino acid residues (Additional file 2: Figure S2). This deleted amino acid sequence partially overlapped with a threoninecontaining binding pocket (A/G-G-X-D/E-T-T/S) regarded as an oxygen-binding motif of cytochrome P450 monooxygenase, required in catalysis [60]. Although in vitro enzyme activity was not examined, the lack of this essential motif is very likely responsible for diminished F3'H enzymatic activity. In addition, CrW exhibits a shorter UV maximum absorption peak-333 nm compared with $346 \mathrm{~nm}$ in $\mathrm{CrV}$-and recovered anthocyanin pigmentation in F3H-overexpressing $\mathrm{CrW}$ petals are pink, a color arising from pelargonidin derivatives that lack B-ring hydroxylation (Additional file 2: Figure S7). These results also indicate the absence of $\mathrm{F}^{\prime} \mathrm{H}$ and $\mathrm{F} 3^{\prime} 5^{\prime} \mathrm{H}$ enzyme activities. It is unlikely, however, that these $F 3^{\prime} H$ and $F 3^{\prime} 5^{\prime} H$ mutations are responsible for white coloration in CrW. For example, deficiency of hydroxylation activity in the anthocyanidin B-ring caused a color shift, but not to white as reported previously [56,59]. How CrW came to possess $F 3^{\prime} H$ and $F 3^{\prime} 5^{\prime} H$ mutations is clearly unknown, but they probably arose in this cultivar during breeding selection for flower colors such as pink, lavender, and blue. Another possibility is that the breeding parents of $\mathrm{CrW}$ contained the latent mutations of these two genes. Torenia plants of the Crown series have various flower colors, and this variation may depend on genotype. In fact, $\mathrm{CrV}$ is heterozygous with respect to these two genes. It is also likely that the other flavonoid biosynthetic genes, besides $F 3^{\prime} H$ and $F 3^{\prime} 5^{\prime} H$, have heterozygous mutations, because torenia is primarily an outcrossing species. Further analysis of other cultivars of the Crown series should also provide clues useful for answering this question.

\section{Conclusions}

In summary, we investigated the causal factor(s) of white flower color in Crown White, a torenia cultivar. Using transient and transgenic approaches, we demonstrated that a novel retrotransposable element (TORE1) inserted into the $5^{\prime}$-upstream region of the $F 3 H$ gene is directly responsible for the colorless flower phenotype. Further investigations will be required to understand the dynamics of TORE1 in the torenia genome. As torenia cultivars have been produced in various flower colors, we expect that additional mutations will soon be uncovered using molecular approaches. We believe that the Crown White cultivar will be useful for genetic engineering of novel flower colors using various plant pigments.

\section{Methods}

\section{Plant materials}

Torenia fournieri plants were used in this study. Whiteflowered Crown White $(\mathrm{CrW})$ and violet-flowered Crown Violet (CrV) cultivars selected from 'Crown Mix' (PanAmerican Seed Company, West Chicago, IL, USA) were kindly provided by Dr. Ryutaro Aida (National Institute of Floricultural Science, Japan). The plants were cultivated in a greenhouse under natural conditions from spring to 
summer. For transformation experiments, in vitro cultured plants were used as described previously [61].

\section{Analysis of pigment components in torenia flower petals} Fully opened flower petals of $\mathrm{CrV}$ and $\mathrm{CrW}$ were used. Flower petals were extracted in $0.1 \% \mathrm{HCl}-$ methanol with gentle shaking overnight at $4^{\circ} \mathrm{C}$. Spectrophotometric analysis was performed using a SpectroMax 190 absorbance microplate reader (Molecular Devices, Sunnyvale, CA, USA). Flavonoid aglycones of anthocyanins and flavones in flower petals were analyzed by high-performance liquid chromatography (HPLC) as described previously [62].

\section{Expression analyses of flavonoid biosynthetic genes}

Total RNA was isolated from opening flowers (stage 4 as defined by Ueyama et al. [9]) using a FastRNA pro GREEN kit (Qbiogene, Irvine, CA, USA). cDNAs were synthesized from total RNA after removal of genomic DNA using a PrimerScript RT reagent kit with gDNA Eraser (Takara Bio, Shiga, Japan). Semi-quantitative RT-PCR analysis was performed using primer sets listed in Additional file 1: Table S1 for nine flavonoid biosynthetic genes from $T$. hybrida: chalcone synthase (CHS, accession no. AB012923), chalcone isomerase (CHI, AB548584), flavanone 3hydroxylase ( $F 3 H, \mathrm{AB} 211958)$, dihydroflavonol 4-reductase (DFR, AB012924), anthocyanidin synthase (ANS, AB044091), flavonoid 3'-hydroxylase ( $F 3^{\prime} H$, AB057672), flavonoid $3^{\prime}, 5^{\prime}$-hydroxylase $\left(F 3^{\prime}, 5^{\prime} H\right.$, AB012925), anthocyanin 5-Oglucosyltransferase (5GT, AB076698), and flavone synthase II (FNSII, AB028152). An actin gene (ACT3, AB330989) was used as an endogenous control. Each 50$\mu \mathrm{L}$ reaction mixture contained $1 \times$ Ex Taq buffer, $200 \mu \mathrm{M}$ dNTPs, $0.5 \mu \mathrm{M}$ of each primer, 5 units Ex Taq polymerase (Takara Bio), and $1 \mu \mathrm{L}$ cDNA template. PCR conditions were as follows: $1 \mathrm{~min} 30 \mathrm{~s}$ at $94^{\circ} \mathrm{C}$, followed by 26 to 34 cycles of $20 \mathrm{~s}$ at $94^{\circ} \mathrm{C}, 40 \mathrm{~s}$ at $55^{\circ} \mathrm{C}$, and $2 \mathrm{~min}$ at $72^{\circ} \mathrm{C}$, and a final extension of $10 \mathrm{~min}$ at $72^{\circ} \mathrm{C}$. The PCR products were separated by electrophoresis on a $1.0 \%$ agarose gel in TAE buffer and stained with ethidium bromide.

Total RNAs $(5 \mu \mathrm{g})$ were subjected to northern blot analysis. Probes for torenia $F 3 H, F 3^{\prime} H$, and $F 3^{\prime} 5^{\prime} H$ were prepared using a PCR-DIG Probe synthesis kit (Roche Diagnostics, Basel, Switzerland) using primers pairs listed in Additional file 1: Table S1. Hybridization and detection were performed using a DIG Nucleic Acid Detection kit (Roche Diagnostics).

\section{Determination of torenia $F 3 H, F 3^{\prime} H$, and $F 3^{\prime} 5^{\prime} H$ gene sequences}

Genomic DNAs were isolated from leaves of each cultivar using a Nucleon PhytoPure kit (GE Healthcare, Little Chalfont, UK). PCR reactions were performed as described above, except that genomic
DNAs were substituted for the cDNA templates. The primer pairs used are listed in Additional file 1: Table S1. The amplified fragments were subcloned into a pCR4TOPO TA cloning vector (Life Technologies, Carlsbad, CA, USA) and sequenced using a BigDye Terminator version 1.1 cycle sequencing kit on an ABI 3130 genetic analyzer (Life Technologies).

\section{Isolation of 5 -upstream regions of the torenia $\mathrm{F} 3 \mathrm{H}$ gene}

The $5^{\prime}$-upstream region of the torenia $F 3 H$ gene was identified using inverse PCR. One microgram of genomic DNA of $\mathrm{CrV}$ was digested with the restriction enzyme Hind III and self-ligated using a Takara ligation kit version 3.0 (Takara Bio). Inverse PCR was performed in $25-\mu \mathrm{l}$ reaction mixtures containing $100 \mathrm{ng}$ ligated genomic DNA, $1 \times L A$ buffer, $2.5 \mathrm{mM}$ $\mathrm{MgCl}_{2}, 400 \mu \mathrm{M}$ dNTPs, $0.2 \mu \mathrm{M}$ of each primer, and 1.25 units of LA Taq polymerase (Takara Bio). The primer sets used are described in Additional file 1: Table S1. Reaction conditions consisted of pre-heating at $94^{\circ} \mathrm{C}$ for $90 \mathrm{~s}, 35$ cycles at $95^{\circ} \mathrm{C}$ for $20 \mathrm{~s}, 60^{\circ} \mathrm{C}$ for $40 \mathrm{~s}$, and $72^{\circ} \mathrm{C}$ for $3 \mathrm{~min}$, and an extension at $72^{\circ} \mathrm{C}$ for $10 \mathrm{~min}$. Amplified fragments of about $5 \mathrm{~kb}$ for $\mathrm{F} 3 \mathrm{H}$ were subcloned and sequenced as described above. The putative transcriptional initiation site was determined by 5'-RACE technology using a GeneRacer kit (Life Technologies). Several primers designed from the sequence of the $5^{\prime}$-upstream region of $\mathrm{CrV}$ were used for PCR amplification of CrW. The PCR products were also subcloned and sequenced as described above.

\section{Southern blot analysis of torenia $F 3 H, F 3^{\prime} H$, and $F 3^{\prime} 5^{\prime} H$ genes and TORE1}

Genomic DNAs $(10 \mu \mathrm{g})$ were digested with Hind III, Eco RI, or Xba I, followed by separation on 1\% agarose gels and transfer to nylon membranes. Coding sequences of $F 3 H, F 3^{\prime} H$, and $F 3^{\prime} 5^{\prime} H$ were used as probes. LTR and gag-pol protein sequences were also used. Hybridization and detection was performed as described previously [34].

\section{Transient expression assays of $\mathrm{F} 3 \mathrm{H}$ promoter activities using Arabidopsis suspension cells}

Arabidopsis thaliana suspension cell line T87 was provided by RIKEN BRC, a participant in the National Bio-Resource Project of the MEXT (Ministry of Education, Culture, Sports, Science and Technology), Japan. Protoplast isolation and transfection experiments for transient expression assays were performed as described by Hartmann et al. [63]. 35SproGtMYB3 and 35Spro-GtbHLH1 vectors were used as effector vectors [33]. An approximately 1-kb long 5'upstream region prior to the translation start site of 
the torenia $F 3 H$ gene was amplified and used as the $T f F 3 H$ promoter. TfF $3 H$ promoters with or without transposon insertions were fused to LUC (firefly luciferase gene). The gentian GtF3H promoter was also used. 35S-RLUC (Renilla luciferase gene) was coinfected as a transformation control. Dual luciferase assays were performed as described previously [64].

\section{Complementation study by transformation of torenia plants}

A binary vector was constructed to express a gentian F3H cDNA (GtF3H-1 [62], accession no. AB193311) in $\mathrm{CrW}$. The plasmid, pSKan-35SGtF3H, was transformed into Agrobacterium tumefaciens strain EHA101. Torenia transformation was performed as described previously [65] using kanamycin as a selection agent. The generated transgenic plants were transferred to an enclosed greenhouse and cultivated until flowering. Northern blot analysis was performed as described above using probes for torenia and gentian $\mathrm{F} 3 \mathrm{H}$.

\section{Additional files}

Additional file 1: Table S1. List of primer sequences used in this study. Additional file 2: Figure S1. Expression analyses of two transcription factor genes in CrV and CrW. RT-PCR analysis of TFMYB1 and TfbHLH1 and the $\beta$-actin gene. Figure $\mathbf{S 2}$. Schematic structure and mutated sequences of ThF3'H and ThF3'5'H genes. (A) The ThF3'H gene consists of three exons and two introns. (B) The ThF $3^{\prime} 5^{\prime} H$ gene consists of two exons and one intron. Figure $\mathbf{S} 3$. Sequence of the $5^{\prime}$-upstream region of $F 3 H$ in $\mathrm{CrW}$. Figure S4. Insertion of the solo-LTR in $5^{\prime}$-upstream region of $F 3 H$ in CrW. Figure S5. Southern blot analysis of $F 3^{\prime} H$ and $F 3^{\prime} 5^{\prime} H$ in $\mathrm{CrV}$ and $\mathrm{CrW}$. Figure S6. Schematic diagram of the genomic structure of the $\mathrm{F} 3 \mathrm{H}$ gene in CrV and CrW. Figure S7. HPLC analysis of flower petal anthocyanidins in GtF3H-overexpressing transgenic CrW. Figure S8. Example of pigment recovery in a CrW petal. (A) Whole flower. (B) Magnification of boxed red area in $\mathrm{A}$.

\section{Abbreviations \\ F3H: Flavanone 3-hydroxylase; F3'H: Flavonoid 3'-hydroxylase; F3'5' H: Flavonoid 3',5'-hydroxylase; LTR: Long terminal repeat; TORE1: Torenia retrotransposon 1.}

\section{Competing interests}

The authors declare that they have no competing interests.

\section{Authors' contributions}

MN conceived and designed the experiments. EY, MS, and KF carried out the experiments. MN and TN also performed some of the experiments. HT helped to analyze pigments. MN supervised the study and wrote the manuscript. HT and TN critically revised the manuscript and completed it. All authors read and approved the final manuscript.

\section{Acknowledgements}

We gratefully thank Dr. Ryutaro Aida (National Institute of Floricultural Science, Japan) for providing us with the torenia materials and the transformation protocol. We also thank Mrs. Akiko Kubota and Mrs. Emiko Chiba, Iwate Biotechnology Research Center, for technical assistance with production of transgenic torenia plants. The work described here was financially supported by Iwate Prefecture and also in part by Grants-in-Aid for Scientific Research from the Japan Society for the Promotion of Science (No. 25660030).

\section{Author details}

'Iwate Biotechnology Research Center, Narita 22-174-4, Kitakami, Iwate 024-0003, Japan. ${ }^{2}$ Department of Biological and Environmental Science, Graduate School of Agriculture, Shizuoka University, 836 Ohya Suruga-ku, Shizuoka 422-8529, Japan.

Received: 27 January 2014 Accepted: 20 March 2014 Published: 2 April 2014

\section{References}

1. Grotewold E: The Science of Flavonoids. London/New York: Springer-Verlag; 2006.

2. Dixon RA, Liu C, Jun JH: Metabolic engineering of anthocyanins and condensed tannins in plants. Curr Opin Biotechnol 2013, 24:329-335.

3. Dixon RA, Pasinetti GM: Flavonoids and isoflavonoids: from plant biology to agriculture and neuroscience. Plant Physiol 2010, 154:453-457.

4. Petroni $\mathrm{K}$, Tonelli $\mathrm{C}$ : Recent advances on the regulation of anthocyanin synthesis in reproductive organs. Plant Sci 2011, 181:219-229.

5. Aida R: Torenia fournieri (torenia) as a model plant for transgenic studies. Plant Biotechnol 2008, 25:541-545.

6. Nishihara M, Shimoda T, Nakatsuka T, Arimura G: Frontiers of torenia research: innovative ornamental traits and study of ecological interaction networks through genetic engineering. Plant Methods 2013, 9:23.

7. Aida R, Yoshida K, Kondo T, Kishimoto S, Shibata M: Copigmentation gives bluer flowers on transgenic torenia plants with the antisense dihydroflavonol-4-reductase gene. Plant Sci 2000, 160:49-56.

8. Nakamura N, Fukuchi-Mizutani M, Fukui Y, Ishiguro K, Suzuki K, Suzuki $H$, Okazaki K, Shibata D, Tanaka Y: Generation of pink flower varieties from blue Torenia hybrida by redirecting the flavonoid biosynthetic pathway from delphinidin to pelargonidin. Plant Biotechnol 2010, 27:375-383.

9. Ueyama Y, Suzuk IK, Fukuchi-Mizutani M, Fukui Y, Miyazaki K, Ohkawa H, Kusumi T, Tanaka Y: Molecular and biochemical characterization of torenia flavonoid 3'- hydroxylase and flavone synthase II and modification of flower color by modulating the expression of these genes. Plant Sci 2002, 163:253-263.

10. Nagira Y, Shimamura K, Hirai S, Shimanuki M, Kodama H, Ozeki Y: Identification and characterization of genes induced for anthocyanin synthesis and chlorophyll degradation in regenerated torenia shoots using suppression subtractive hybridization, CDNA microarrays, and RNAi techniques. J Plant Res 2006, 119:217-230

11. Sasaki K, Aida R, Yamaguchi H, Shikata M, Niki T, Nishijima T, Ohtsubo N: Functional divergence within class B MADS-box genes TfGLO and TfDEF in Torenia fournieri Lind. Mol Genet Genomics 2010, 284:399-414.

12. Sasaki K, Yamaguchi H, Aida R, Shikata M, Abe T, Ohtsubo N: Mutation in Torenia fournieri Lind. UFO homolog confers loss of TfLFY interaction and results in a petal to sepal transformation. Plant $J$ 2012, 71:1002-1014.

13. Narumi T, Aida R, Niki T, Nishijima T, Mitsuda N, Hiratsu K, Ohme-Takagi M, Ohtsubo N: Chimeric AGAMOUS repressor induces serrated petal phenotype in Torenia fournieri similar to that induced by cytokinin application. Plant Biotechnol 2008, 25:45-53.

14. Narumi T, Aida R, Koyama T, Yamaquchi H, Sasaki K, Shikata M, Nakayama M, Ohme-Takagi M, Ohtsubo N: Arabidopsis chimeric TCP3 repressor produces novel floral traits in Torenia fournieri and Chrysanthemum morifolium. Plant Biotechnol 2011, 28:131-140.

15. Takeuchi H, Higashiyama T: A species-specific cluster of defensin-like genes encodes diffusible pollen tube attractants in Arabidopsis. PLOS Biol 2012, 10:e1001449

16. Suzuki $K$, Xue $H$, Tanaka Y, Fukui $Y$, Fukuchi-Mizutani M, Murakami $Y$, Katsumoto Y, Tsuda S, Kusumi T: Flower color modifications of Torenia hybrida by cosuppression of anthocyanin biosynthesis genes. Mol Breed 2000, 6:239-246.

17. Fukusaki E, Kawasaki K, Kajiyama S, An Cl, Suzuki K, Tanaka Y, Kobayashi A: Flower color modulations of Torenia hybrida by downregulation of chalcone synthase genes with RNA interference. J Biotechnol 2004, 111:229-240.

18. Nakamura N, Fukuchi-Mizutani M, Miyazaki K, Suzuki K, Tanaka Y: RNAi suppression of the anthocyanidin synthase gene in Torenia hybrida yields white flowers with higher frequency and better stability than antisense and sense suppression. Plant Biotechnol 2006, 23:13-17. 
19. Ono E, Fukuchi-Mizutani M, Nakamura N, Fukui Y, Yonekura-Sakakibara K, Yamaguchi M, Nakayama T, Tanaka T, Kusumi T, Tanaka Y: Yellow flowers generated by expression of the aurone biosynthetic pathway. Proc Nat Acad Sci U S A 2006, 103:11075-11080.

20. Gerats AG, Huits H, Vrijlandt E, Marana C, Souer E, Beld M: Molecular characterization of a nonautonomous transposable element (dTph1) of petunia. Plant Cell 1990, 2:1121-1128.

21. Clegg MT, Durbin ML: Flower color variation: a model for the experimental study of evolution. Proc Natl Acad Sci U S A 2000, 97:7016-7023.

22. Martin C, Prescott A, Mackay S, Bartlett J, Vrijlandt E: Control of anthocyanin biosynthesis in flowers of Antirrhinum majus. Plant J 1991, 1:37-49.

23. Hudson A, Critchley J, Erasmus Y: The genus Antirrhinum (snapdragon): a flowering plant model for evolution and development. CSH Protocols 2008, 2008:pdb emo100

24. Hoshino A, Abe Y, Saito N, Inagaki Y, lida S: The gene encoding flavanone 3hydroxylase is expressed normally in the pale yellow flowers of the Japanese morning glory carrying the speckled mutation which produce neither flavonol nor anthocyanin but accumulate chalcone, aurone and flavanone. Plant Cell Physiol 1997, 38:970-974.

25. lida S, Morita Y, Choi JD, Park Kl, Hoshino A: Genetics and epigenetics in flower pigmentation associated with transposable elements in morning glories. Adv Biophys 2004, 38:141-159.

26. Inagaki $Y$, Hisatomi $Y$, Suzuki $T$, Kasahara $K$, lida S: Isolation of a Suppressor-mutator/Enhancer-like transposable element, Tpn1, from Japanese morning glory bearing variegated flowers. Plant Cell 1994, 6:375-383.

27. Hoshino A, Park Kl, lida S: Identification of $r$ mutations conferring white flowers in the Japanese morning glory (Ipomoea nil). J Plant Res 2009, 122:215-222.

28. Matsubara K, Kodama H, Kokubun H, Watanabe H, Ando T: Two novel transposable elements in a cytochrome $\mathrm{P} 450$ gene govern anthocyanin biosynthesis of commercial petunias. Gene 2005, 358:121-126.

29. Itoh Y, Higeta D, Suzuki A, Yoshida H, Ozeki Y: Excision of transposable elements from the chalcone isomerase and dihydroflavonol 4-reductase genes may contribute to the variegation of the yellow-flowered carnation (Dianthus caryophyllus). Plant Cell Physiol 2002, 43:578-585.

30. Momose M, Nakayama M, Itoh $Y$, Umemoto N, Toguri T, Ozeki Y: An active $h A T$ transposable element causing bud mutation of carnation by insertion into the flavonoid 3'-hydroxylase gene. Mol Genet Genomics 2013, 288:175-184.

31. Nishizaki Y, Matsuba Y, Okamoto E, Okamura M, Ozeki Y, Sasaki N: Structure of the acyl-glucose-dependent anthocyanin 5-O-glucosyltransferase gene in carnations and its disruption by transposable elements in some varieties. Mol Genet Genomics 2011, 286:383-394.

32. Nakatsuka T, Nishihara M, Mishiba K, Yamamura S: Two different mutations are involved in the formation of white-flowered gentian plants. Plant Sci 2005, 169:949-958

33. Nakatsuka T, Haruta KS, Pitaksutheepong C, Abe Y, Kakizaki Y, Yamamoto K, Shimada N, Yamamura S, Nishihara M: Identification and characterization of R2R3-MYB and bHLH transcription factors regulating anthocyanin biosynthesis in gentian flowers. Plant Cell Physiol 2008, 49:1818-1829.

34. Nishihara M, Hikage T, Yamada E, Nakatsuka T: A single-base substitution suppresses flower color mutation caused by a novel miniature invertedrepeat transposable element in gentian. Mol Genet Genomics 2011, 286:371-382.

35. Nishijima T, Morita Y, Sasaki K, Nakayama M, Yamaguchi H, Ohtsubo N, Niki T, Niki T: A torenia (Torenia fournieri Lind. ex Fourn.) Novel mutant 'Flecked' produces variegated flowers by insertion of a DNA transposon into an R2R3-MYB gene. J Japan Soc Hort Sci 2013, 39:39-50

36. Xiao $H$, Jiang $N$, Schaffner E, Stockinger EJ, van der Knaap E: A retrotransposon-mediated gene duplication underlies morphological variation of tomato fruit. Science 2008, 319:1527-1530.

37. Rico-Cabanas L, Martinez-Izquierdo JA: CIRE1, a novel transcriptionally active Ty1-copia retrotransposon from Citrus sinensis. Mol Genet Genomics 2007, 277:365-377

38. Nakatsuka T, Yamada E, Saito M, Fujita K, Nishihara M: Heterologous expression of gentian MYB1R transcription factors suppresses anthocyanin pigmentation in tobacco flowers. Plant Cell Rep 2013, 32:1925-1937.

39. Koes R, Verweij W, Quattrocchio F: Flavonoids: a colorful model for the regulation and evolution of biochemical pathways. Trends Plant Sci 2005 10:236-242.
40. Britsch L, Ruhnau-Brich B, Forkmann G: Molecular cloning, sequence analysis, and in vitro expression of flavanone $3 \beta$-hydroxylase from Petunia hybrida. J Biol Chem 1992, 267:5380-5387.

41. van Houwelingen A, Souer E, Spelt K, Kloos D, Mol J, Koes R: Analysis of flower pigmentation mutants generated by random transposon mutagenesis in Petunia hybrida. Plant J 1998, 13:39-50.

42. Zabala G, Vodkin LO: The wp mutation of Glycine max carries a genefragment-rich transposon of the CACTA superfamily. Plant Cell 2005, 17:2619-2632.

43. Dedio J, Saedler H, Forkmann G: Molecular cloning of the flavanone $3 \beta-$ hydroxylase gene (FHT) from carnation (Dianthus caryophyllus) and analysis of stable and unstable FHT mutants. Theor Appl Genet 1995, 90:611-617.

44. Kumar A, Bennetzen JL: Plant retrotransposons. Annu Rev Genet 1999 33:479-532

45. Casacuberta JM, Santiago N: Plant LTR-retrotransposons and MITEs: control of transposition and impact on the evolution of plant genes and genomes. Gene 2003, 311:1-11.

46. Huang CR, Burns KH, Boeke JD: Active transposition in genomes. Annu Rev Genet 2012, 46:651-675.

47. Rebollo R, Horard B, Hubert B, Vieira C: Jumping genes and epigenetics: Towards new species. Gene 2010, 454:1-7.

48. Oliver KR, McComb JA, Greene WK: Transposable elements: powerful contributors to angiosperm evolution and diversity. Genome Biol Evol 2013, 5:1886-1901

49. Grandbastien M, Casacuberta J: Plant transposable elements. Impact on genome structure and function. Springer Berlin Heidelberg: Springer; 2012.

50. Uchiyama T, Fujino K, Ogawa T, Wakatsuki A, Kishima Y, Mikami T, Sano Y: Stable transcription activities dependent on an orientation of Tam3 transposon insertions into Antirrhinum and yeast promoters occur only within chromatin. Plant Physiol 2009, 151:1557-1569.

51. McClelland M, Nelson M, Raschke E: Effect of site-specific modification on restriction endonucleases and DNA modification methyltransferases. Nucleic Acids Res 1994, 22:3640-3659.

52. Jones $\mathrm{AL}$, Thomas $\mathrm{CL}$, Maule AJ: De novo methylation and co-suppression induced by a cytoplasmically replicating plant RNA virus. EMBO J 1998, 17:6385-6393.

53. Vitte C, Panaud O: Formation of solo-LTRs through unequal homologous recombination counterbalances amplifications of LTR retrotransposons in rice Oryza sativa L. Mol Biol Evol 2003, 20:528-540.

54. Devos KM, Brown JK, Bennetzen JL: Genome size reduction through illegitimate recombination counteracts genome expansion in Arabidopsis. Genome Res 2002, 12:1075-1079.

55. Ma J, Bennetzen JL: Rapid recent growth and divergence of rice nuclear genomes. Proc Natl Acad Sci U S A 2004, 101:12404-12410.

56. Toda K, Yang D, Yamanaka N, Watanabe S, Harada K, Takahashi R: A singlebase deletion in soybean flavonoid 3'-hydroxylase gene is associated with gray pubescence color. Plant Mol Biol 2002, 50:187-196.

57. Takahashi R, Githiri SM, Hatayama K, Dubouzet EG, Shimada N, Aoki T, Ayabe S, Iwashina T, Toda K, Matsumura H: A single-base deletion in soybean flavonol synthase gene is associated with magenta flower color. Plant Mol Biol 2007, 63:125-135.

58. Morita $Y$, Hoshino A, Kikuchi $Y$, Okuhara H, Ono E, Tanaka Y, Fukui Y, Saito N, Nitasaka E, Noguchi $H$, lida S: Japanese morning glory dusky mutants displaying reddish-brown or purplish-gray flowers are deficient in a novel glycosylation enzyme for anthocyanin biosynthesis, UDP-glucose: anthocyanidin 3-O-glucoside-2"-O-glucosyltransferase, due to 4-bp insertions in the gene. Plant J 2005, 42:353-363.

59. Hoshino A, Morita Y, Choi JD, Saito N, Toki K, Tanaka Y, lida S: Spontaneous mutations of the flavonoid 3'-hydroxylase gene conferring reddish flowers in the three morning glory species. Plant Cell Physiol 2003, 44:990-1001.

60. Durst F, Nelson DR: Diversity and evolution of plant P450 and P450reductases. Drug Metabol Drug Interact 1995, 12:189-206.

61. Shimoda T, Nishihara M, Ozawa R, Takabayashi J, Arimura G: The effect of genetically enriched $(E)$ - $\beta$-ocimene and the role of floral scent in the attraction of the predatory mite Phytoseiulus persimilis to spider miteinduced volatile blends of torenia. New Phytol 2012, 193:1009-1021.

62. Nakatsuka T, Nishihara M, Mishiba K, Yamamura S: Temporal expression of flavonoid biosynthesis-related genes regulates flower pigmentation in gentian plants. Plant Sci 2005, 168:1309-1318.

63. Hartmann U, Sagasser M, Mehrtens F, Stracke R, Weisshaar B: Differential combinatorial interactions of cis-acting elements recognized by R2R3- 
MYB, BZIP, and BHLH factors control light-responsive and tissue-specific activation of phenylpropanoid biosynthesis genes. Plant Mol Biol 2005, 57:155-171.

64. Nakatsuka T, Saito M, Yamada E, Fujita K, Kakizaki Y, Nishihara M: Isolation and characterization of GtMYBP3 and GtMYBP4, orthologues of R2R3MYB transcription factors that regulate early flavonoid biosynthesis, in gentian flowers. J Exp Bot 2012, 63:6505-6517.

65. Aida R: A protocol for transformation of Torenia. Methods Mol Biol 2012, 847:267-274.

doi:10.1186/1471-2229-14-86

Cite this article as: Nishihara et al:: Molecular characterization of

mutations in white-flowered torenia plants. BMC Plant Biology 2014 14:86

\section{Submit your next manuscript to BioMed Central} and take full advantage of:

- Convenient online submission

- Thorough peer review

- No space constraints or color figure charges

- Immediate publication on acceptance

- Inclusion in PubMed, CAS, Scopus and Google Scholar

- Research which is freely available for redistribution

Submit your manuscript at www.biomedcentral.com/submit

C Biomed Central 